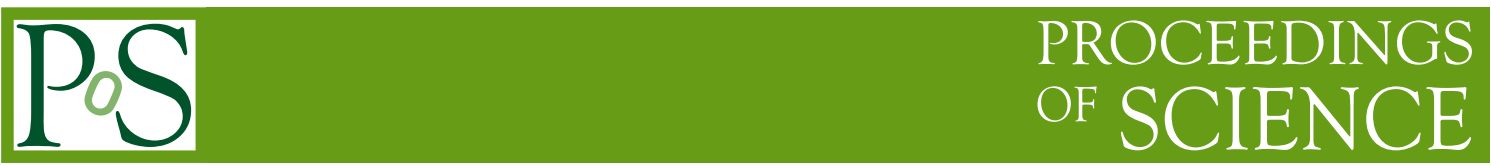

\title{
GX 339-4: spectral variability of the hard X-ray emission
}

\author{
Robert Droulans*, Renaud Belmont, Julien Malzac and Elisabeth Jourdain \\ Université de Toulouse ; UPS ; CESR ; 9 avenue du Colonel Roche, F-31028 Toulouse, France \\ CNRS ; UMR5187; F-31028 Toulouse, France \\ E-mail: droulans@cesr.fr
}

\begin{abstract}
We study the high energy emission of the Galactic black hole candidate GX 339-4 using INTEGRAL/SPI and simultaneous RXTE/PCA data. By the end of January 2007, when it reached its peak luminosity in hard X-rays, the source was in a very bright low hard state. The SPI data from this period show a good signal to noise ratio, allowing a detailed study of the spectral energy distribution up to several hundred $\mathrm{keV}$. As a main result, we report on the detection of a variable hard spectral feature $(\geq 150 \mathrm{keV})$ which represents a significant excess with respect to the cutoff power law shape of the spectrum. The SPI data suggest that the intensity of this feature is positively correlated with the $25-50 \mathrm{keV}$ luminosity of the source and the associated variability time scale is shorter than 7 hours. The simultaneous $P C A$ data, however, show no significant change in the spectral shape, indicating that the source is not undergoing a canonical state transition. We present the results of quantitative model fitting of the joint X-ray spectra with the new Comptonization code веLм, which self-consistently accounts for the presence of a magnetic field within the Comptonizing electron plasma.
\end{abstract}

The Extreme sky: Sampling the Universe above $10 \mathrm{keV}$

October 13-17 2009

Otranto (Lecce) Italy

${ }^{*}$ Speaker. 


\section{Introduction}

Black hole binaries (BHBs) are powerful engines producing high-energy radiation up to the $\gamma$-ray domain. From an observational perspective, they appear in two canonical spectral states, namely, low/hard (LHS) and high/soft (HSS) [1]. According to a popular scenario, these states can be explained through changes in the geometry of the accretion flow [4].

In the HSS, there is solid evidence that a geometrically thin accretion disc extends down to the last stable orbit. In the LHS, however, the weakness of the thermal component is generally interpreted as a consequence of a truncated accretion disk. In its inner parts, the standard optically thick disk is replaced by a hot, advection dominated accretion flow (ADAF; $[13,11]$ ) which naturally forms the quasi thermal electron distributions that are required to explain the typical hard state spectra. However, a number of recent observations seem to conflict with this picture. First, it is not completely clear whether in the LHS the disc receedes or not. (see e.g. [3] and [12]). Second, the proton temperature in some BHBs is likely to be too low to be consistent with the predictions of typical two-temperature flow solutions [9] (hereafter MB09).

As an alternative to the ADAF-like models, the corona in the LHS could as well be powered by the same non-thermal mechanisms that are believed to accelerate the electrons in the HSS, i.e. diffusive shock acceleration or magnetic reconnection [8]. If this is the case, it is important to understand the origin of the observed differences in the Comptonising electron distributions.

In this context, MB09 showed that the steady state electron distribution can appear thermal even if acceleration mechanisms are purely non-thermal. In addition to $e$-e Coulomb interactions, they studied the thermalizing effects of the magnetic field, since it was pointed out by Ghisellini et al. [6] that the very fast emission and absorption of synchrotron photons (the so-called 'synchrotron boiler effect') is able to thermalize the electron distribution in a few light-crossing times. Using BELM, a new code which includes this effect [2], MB09 proposed a global scenario which qualitatively explains the spectral states observed in the prototyicpal black hole binary Cyg X-1.

Here, we present related issues in the case of GX 339-4. We use observations with SPI/INTEGRAL and $P C A / R X T E$ to study the spectral energy distribution of the bright LHS from the 2007 outburst (for more details see Droulans et al. [5]).

\section{SPI light-curve and high energy spectra}

Fig. 1 shows the $25-50 \mathrm{keV}$ SPI light curve from GX 339-4 obtained during INTEGRAL revolution 525. Each bin represents one science-window, which corresponds to a time scale of about $40 \mathrm{~min}$. The light-curve reveals minor variability (on a time scale of hours, or less) around the average flux value $\left\langle F>=657.1 \pm 3.8 \mathrm{mCrab}\right.$. We fixed two arbitrary flux bounds $\left(F_{\text {low }}=640 \mathrm{mCrab}\right.$ and $\left.F_{\text {high }}=670 \mathrm{mCrab}\right)$ and grouped the science-windows where $F_{\text {scw }}<F_{\text {low }}$ and $F_{\text {scw }}>F_{\text {high }}$, respectively. For each of the two resulting data subsets, color-coded in blue and red (cf. Fig. 1) and referred to as low and high respectively, we then produced the averaged $25-500 \mathrm{keV}$ spectrum. The science-windows plotted in black were not considered in the present work. We fitted both spectra with a simple model consisting of a power law with a high-energy cutoff. As the SPI data alone do not allow to simultaneously constrain the photon index and the cutoff energy, we fixed the former to $\Gamma=1.3$ and left the latter free to vary. The model provides a good fit to the 


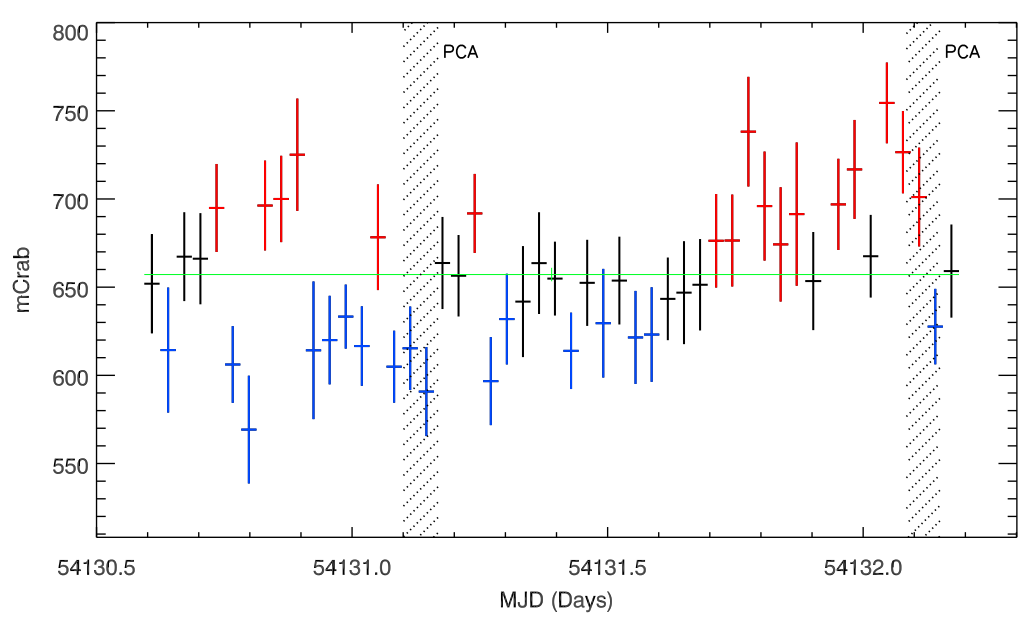

Figure 1: $25-50 \mathrm{keV}$ SPI light curve from revolution 525 . The pointings selected for the high and low flux data sets are shown in red and blue, respectively. The shaded areas indicate the time periods of the simultaneous $P C A$ observations.

low spectrum $\left(\chi^{2} / 22=0.75\right)$ and we infer a significant cutoff at $E_{c}=58.6 \pm 2.2 \mathrm{keV}$. For the high spectrum, the marked curvature around $40 \mathrm{keV}$ still suggests the presence of a cutoff energy (fitted at $E_{c}=56.2 \pm 2.1 \mathrm{keV}$ ). With respect to the model, however, we observe a significant excess above $150 \mathrm{keV}$, leading to an unacceptable quality of the fit $\left(\chi^{2} / 28=1.82\right)$. We conclude that both SPI spectra essentially differ in terms of the highest energy emission $(>150 \mathrm{keV})$ and rename them to cutoff and excess, respectively.

\section{Physical analysis}

We will first introduce the general physical framework used to interpret the joint SPI/PCA spectra and then focus on two specific situations in which the effects of a given parameter are emphasized.

\subsection{General model}

The high energy source in GX 339-4 is modeled by a spherical, magnetized, fully ionized proton-electron/positron plasma of radius $R$. The emission of the plasma is derived by self-consistent computations of the equilibrium electron distribution accounting for Compton scattering, synchrotron emission/absorption and pair production/annihilation. The Thomson optical depth of the plasma is given by $\tau_{T}=\tau_{\text {ion }}+\tau_{\text {pair }}$, where $\tau_{\text {ion }}$ represents the optical depth of ionization electrons and $\tau_{\text {pair }}$ is the opacity that arises from pair production.

The plasma properties essentially depend on the magnetic field strength as well as on the power supplied by external sources. The tangled magnetic field strength is parameterized by the magnetic compactness $l_{B}=\frac{\sigma_{T}}{m_{e} c^{2}} R \frac{B^{2}}{8 \pi}$. Energy injection is quantified by the radiative compactness $l=\frac{\sigma_{T}}{m_{e} c^{3}} \frac{L}{R}$, where $L$ is the power supplied to the plasma and $\sigma_{T}$ the Thomson cross-section. We assume only one possible channel for providing energy to the coupled electron-photon system, namely through non-thermal electron acceleration $\left(l=l_{n t h}\right)$. The acceleration processes are mimicked by continuous electron injection at a power law rate with index $\Gamma_{i n j}\left(\right.$ i.e. $n(\gamma) \propto \gamma^{-\Gamma_{i n j}}$ ) and Lorentz factors 


\begin{tabular}{ccccccccc}
\hline \hline \multirow{2}{*}{ Model } & Spec & $\Gamma_{i n j}$ & $\gamma_{\max }$ & $l_{B}$ & $\tau_{\text {ion }}$ & $\Omega / 2 \pi$ & $\xi$ & $\chi_{\nu}^{2}(\mathrm{dof})$ \\
\hline \hline \multirow{2}{*}{ ICM1 } & cutoff & $3.47_{-0.31}^{+0.66}$ & $1000^{\star}$ & $742_{-142}^{+201}$ & $2.26^{ \pm 0.18}$ & $0.43^{ \pm 0.05}$ & $299_{-97}^{+122}$ & $0.96(68)$ \\
& excess & $2.50_{-0.08}^{+0.32}$ & $1000^{\star}$ & $25.2^{ \pm 3.6}$ & $2.14_{-0.17}^{+0.14}$ & $0.44_{-0.03}^{+0.06}$ & $258_{-84}^{+109}$ & $1.06(74)$ \\
\multirow{2}{*}{ ICM2 } & cutoff & $2.5^{\star}$ & $15.2_{-1.3}^{+5.5}$ & $283_{-36}^{+43}$ & $2.30_{-0.16}^{+0.17}$ & $0.43_{-0.03}^{+0.04}$ & $301_{-100}^{+123}$ & $0.96(68)$ \\
& excess & $2.5^{\star}$ & $187_{-105}^{+111}$ & $27.3_{-3.4}^{+4.0}$ & $2.49^{ \pm 0.18}$ & $0.39_{-0.04}^{+0.04}$ & $310_{-126}^{+155}$ & $0.92(74)$ \\
\hline
\end{tabular}

Table 1: Best fit parameters of the joint SPI and PCA spectra. Frozen parameters are indicated by a star $\left(^{\star}\right)$ next to their value.

ranging from $\gamma_{\min }$ to $\gamma_{\max }$.

For a given source flux $F$, the radiative compactness can be estimated by the formula:

$$
l=100\left(\frac{F}{F_{0}}\right)\left(\frac{d}{8 k p c}\right)^{2}\left(\frac{13 M_{\odot}}{M}\right)\left(\frac{30 R_{g}}{R}\right)
$$

where $M$ is the mass of the black hole and $d$ the distance to the source. Here we expressed $l$ in terms of $F_{0}=2.6 \times 10^{-8} \mathrm{erg} \mathrm{s}^{-1} \mathrm{~cm}^{-2}$, the observed bolometric flux of GX 339-4. Assuming a spherical $\mathrm{X}$-ray corona of radius $R=30 \mathrm{R}_{G}$, a black hole mass of $M=13 \mathrm{M}_{\odot}$ and a distance of $d=8 \mathrm{kpc}$, this leads to a compactness of $l \sim 100$. The model also accounts for relativistically blurred Compton reflection from a potentially ionized accretion disc [7] at a fixed inclination angle of $50^{\circ}$.

To calculate the model spectra, we used the new versatile Comptonization code вELм [2]. The stated errors are at the 90 per cent confidence level $\left(\Delta \chi^{2}=2.7\right)$ and the fitting results are summarized in Table 1.

\subsection{Variable injection index}

First, we investigate the effects of a variable spectral index of the injected electron distribution. In this model, referred to as ICM1 (for Internal Comptonization Model 1$)$, we set $\left(\gamma_{\min }, \gamma_{\max }\right)=$ $(1.3,1000)$ and use Eq. 3.1 to fix $l_{\text {nth }}=100$. For the cutoff spectrum, we obtain a good fit to the data $\left(\chi^{2} / 68=0.96\right)$ and infer a spectral index of $\Gamma_{i n j}=3.47_{-0.31}^{+0.66}$. The magnetic compactness is fitted at $l_{B}=742_{-142}^{+201}$ and the plasma is found to be of moderate optical thickness, with fitted $\tau_{\text {ion }}=2.26 \pm$ 0.18 . Due to the fast synchrotron cooling of the initially small number of high energy particles, the produced pair yield is negligible. The data strongly require ionized Compton reflection, with a fitted amplitude and ionization factor of $\Omega / 2 \pi=0.43 \pm 0.05$ and $\xi=299_{-97}^{+122}$, respectively. For the excess spectrum, we obtain again a good description of the data. The best fit yields $\chi^{2} / 74=1.06$ and the high energy data constrain the injection index to $\Gamma_{i n j}=2.50_{-0.08}^{+0.32}$. We find a magnetic compactness of $l_{B}=25.2 \pm 3.6$, which is almost a factor 30 lower than the value derived from the cutoff spectrum. The Thomson optical depth of ionization electrons and the reflection parameters, however, are consistent with the results obtained for the cutoff spectrum.

\subsection{Variable maximum particle energy}

In a second model (ICM2), we investigate the effects of a varying maximum energy of the accelerated electrons. We keep $\gamma_{\min }=1.3$ and $l_{n t h}=100$ but fix the spectral index to $\Gamma_{i n j}=2.5$. For 

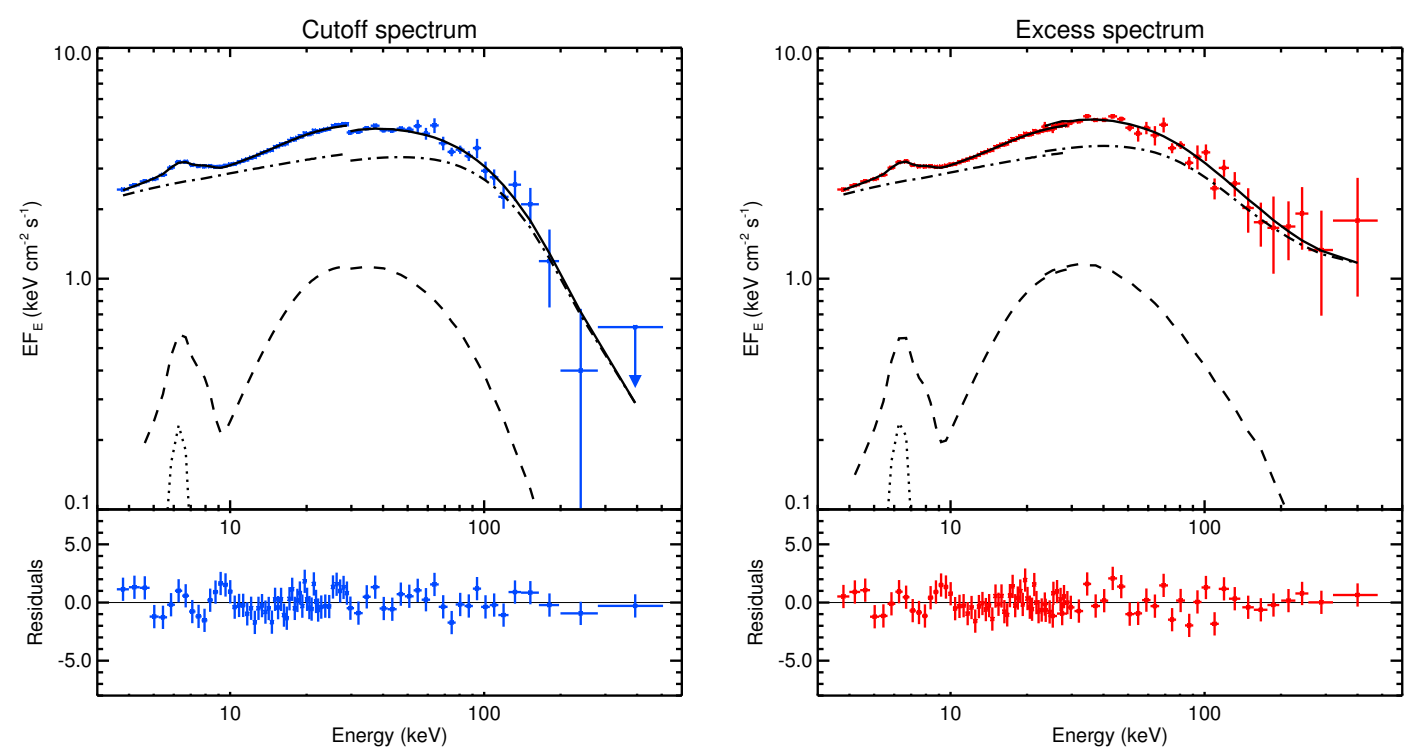

Figure 2: Joint SPI and PCA spectra fitted with the ICM2, the cutoff spectrum in red, the excess spectrum in blue. The solid line is the total model, the dot-dashed line represents the SSC component while the dashed and dotted lines show the reflection component and the iron line emission, respectively. The error bars are given at the $1 \sigma$ level.

the cutoff spectrum, we obtain a good fit to the data $\left(\chi^{2} / 68=0.96\right.$; shown in Fig. 2 left). This shows that the effects of a very soft injection slope can be mimicked by a much harder distribution, but truncated at a certain particle energy. We find $\gamma_{\max }=15.2_{-1.3}^{+5.5}$ and infer a magnetic compactness of $l_{B}=283_{-36}^{+43}$. The other parameters are consistent with the results obtained in the previous paragraph. For the excess spectrum, the injection index is frozen to the best fit value obtained with the ICM1. Nevertheless, allowing for $\gamma_{\max }$ to vary, the fit may be improved considerably. For $\gamma_{\max }=187_{-105}^{+111}$, we obtain the best description of the excess spectrum, with a reduced $\chi^{2}$ of $\chi^{2} / 74=0.92$ (cf. Fig. 2 right). The magnetic compactness remains equal to the value inferred with the ICM1, namely $l_{B}=27.3_{-3.4}^{+4.0}$. The opacity and reflection parameters are also consistent with the previously obtained results, showing that these values are robust (cf. Table 1).

\section{Discussion and Conclusion}

We presented an analysis of the high energy emission of GX 339-4 in a luminous hard state. With respect to the standard cutoff shape of the hard state spectrum, the $25-500 \mathrm{keV}$ INTEGRAL/SPI data revealed the appearance of a variable high energy excess. The intensity of this hard excess seems to be positively correlated with the $25-50 \mathrm{keV}$ luminosity and the associated time scale is shorter than 7 hours.

Using the new code BeLm [2], we showed that the hard X-ray behavior of GX 339-4 in the bright LHS can be explained by assuming pure non-thermal electron acceleration and subsequent Comptonization of the self-consistently produced synchrotron photons. The model requires no incident radiation from the accretion disc and assumes constant power injection into the magnetized corona. A transition between the two spectra can be explained by the variations of only two parameters. In 
any case, the magnetic compactness $l_{B}$ needs to be variable, along with either the injection slope (in the ICM1) or the maximum energy of the accelerated particles (in the ICM2). All other fit parameters are found to remain constant within the 90 per cent confidence errors.

Even though the nature of the acceleration mechanism remains unclear, the inferred parameter variations are plausible. Indeed, physical changes (e.g. the Mach number or the acceleration geometry) within the turbulent medium may explain the variations of $\Gamma_{i n j}$ and/or $\gamma_{\max }$. However, the ICM2 is more likely because, regardless of the exact nature of the acceleration mechanism, a change of the magnetic field intensity is expected to have an impact on the maximum energy of the accelerated electrons. In the framework of diffusive shock acceleration, it has been shown that when synchrotron losses are included, the maximum Lorentz factor of the accelerated relativistic electrons satisfies $\gamma_{\max } \propto 1 /\left(K B^{2}\right)$, where $K$ is the diffusion coefficient (see e.g. [14] or [10]). If $K$ is constant, this predicts a maximum energy of $\gamma_{\max } \propto 1 / B^{2}$. However, depending on the assumptions made to describe the acceleration mechanism, the diffusion coefficient can depend both on the particle energy and the magnetic field strength: $K(\gamma, B)$. From the best fits obtained with the ICM2, we find that the dependence between $\gamma_{\max }$ and $B$ is approximately $\gamma_{\max } \propto 1 / B^{2}$.

In conclusion, the ICM2 provides the framework for a simple, physically motivated interpretation of the data, showing that the spectral variability could be triggered by the variation of only one single parameter, that is to say the magnetic field intensity. If the fitted average spectra provide a good estimate of the individual spectra from the single science windows, our analysis shows that the time scale of this evolution is at most of the order of hours. The viscous time scale of the accretion disk at a radius of $30 R_{G}$ depends on the disk parameters, but is at most of the order of minutes. Therefore, even if the geometry of the X-ray emitting region and its dynamical evolution are still uncertain, global changes on time scales of hours are not unrealistic.

\section{References}

[1] T. Belloni, J. Homan, P. Casella et al., A\&A 440, 207-222 (2005).

[2] R. Belmont, J. Malzac, and A. Marcowith, A\&A 491, 617-631 (2008).

[3] C. Cabanac, R. P. Fender, R. J. H. Dunn, and E. G. Körding, MNRAS 396, 1415-1440 (2009).

[4] C. Done, M. Gierliński, and A. Kubota, A\&AR 15, 1-66 (2007).

[5] R. Droulans, R. Belmont, J. Malzac and E. Jourdain, submitted to ApJ.

[6] G. Ghisellini, F. Haardt, and R. Svensson, MNRAS 297, 348-354 (1998).

[7] P. Magdziarz and A. A. Zdziarski, MNRAS 273, 837-848 (1995).

[8] J. Malzac, Ap\&SS 311, 149-159 (2007).

[9] J. Malzac and R. Belmont, MNRAS 392, 570-589 (2009).

[10] A. Marcowith and J. G. Kirk, A\&A 347, 391-400 (1999).

[11] R. Narayan and I. Yi, ApJL 428, L13-L16 (1994).

[12] R. C. Reis, A. C. Fabian, R. R. Ross et al. MNRAS 387, 1489-1498 (2008).

[13] S. L. Shapiro, A. P. Lightman, and D. M. Eardley, ApJ 204, 187-199 (1976).

[14] G. M. Webb, L. O. Drury, and P. Biermann, A\&A 137, 185-201 (1984). 